\title{
Principes d'une exception qui ne devrait pas l'être...
}

\author{
Emmanuel Escard ${ }^{\mathrm{a}}$, Isabelle Rinaldi Baud ${ }^{\mathrm{b}}$ \\ a Médecin adjoint responsable, Unité interdisciplinaire de médecine et de prévention de la violence (UIMPV), Département de médecine communautaire, \\ de premier recours et des urgences, Hôpitaux Universitaires de Genève HUG \\ ${ }^{b}$ Assistante sociale, thérapeute de couple et de famille, UIMPV, Département de médecine communautaire, de premier recours et des urgences, HUG
}

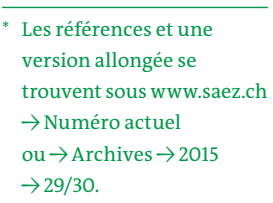

Le monde a des problèmes, mais les universités ont des départements.

(Brewer GD, 1999)

Notre réflexion se fonde sur une pratique de l'interdisciplinarité dans une Unité de médecine et de prévention de la violence, intégrée au sein d'un Département de médecine communautaire, de premier recours et des urgences des Hôpitaux Universitaires de Genève. Cette consultation existe depuis 1997, alors que l'OMS avait déclaré que les violences étaient une priorité de santé publique, et que les professionnels de santé devaient se former à la détection et à la prise en charge large de ces problématiques. Elle a notamment pour mission d'aider les personnes confrontées à des situations de violence quel que soit leur rôle, la nature des violences et le contexte de survenue. Comme les comportements violents sont d'origine multifactorielle et éclairés par différentes disciplines, le parti a été pris dès le départ d'adopter une approche interdisciplinaire avec des évolutions permanentes de la pratique clinique.

Dix-huit ans après, nous constatons que la pratique interdisciplinaire reste rare dans les hôpitaux et les soins ambulatoires et que les "expériences» de cette stratégie de gestion de la complexité restent limitées, tout en

\section{Résumé}

Les violences sont un problème majeur de santé publique qui nécessite une approche interdisciplinaire. Leur prise en charge implique d'être à l'aise avec le complexe, le global, le multidimensionnel et les aspects contextuels et systémiques. C'est aussi avoir la connaissance pertinente pour les affronter, et être ouvert aux incertitudes et à l'inattendu. Pratiquer dans le domaine de la médecine des violences implique d'adapter son cadre éthique de travail, en associant engagement, rigueur, critique, modestie et humanisme, et une recherche poussée du sens et de la coopération avec les patients, leurs proches, les collègues et intervenants du réseau. Une place de choix doit être donnée à l'articulation entre la responsabilité éthique et la narration dans cette activité clinique. Ce mode d'exercice est passionnant mais contraignant, et devrait plus fréquemment s'appliquer en médecine pour d'autres problématiques. étant régulièrement citées et suscitant de l'intérêt dans différents milieux.

Nous proposons donc de repréciser le paradigme de l'interdisciplinarité et quelques règles de bonnes pratiques la concernant que nous essayons d'appliquer.

\section{Pourquoi une approche interdisciplinaire?}

La pratique habituelle en médecine, bien qu'elle soit un art portant sur des êtres humains complexes, est celle d'un travail intra- ou pluridisciplinaire. L'approche interdisciplinaire, elle, est fondée sur le décloisonnement des disciplines, chaque discipline associée apportant savoirs et méthodes tout en gardant sa spécificité, et participant à un projet collectif. Les professionnels échangent entre eux, collaborent et enrichissent leurs compétences pour répondre aux besoins de la compréhension et de l'action. Ils refusent le savoir émietté et tendent à relier les connaissances grâce à la pensée complexe qui englobe au lieu de séparer. Cette pratique du réel dans notre unité a été la source du développement de différents instruments et méthodes de travail pour les professionnels qui ont été précédemment exposés [1-4]*.

L'approche interdisciplinaire pose deux questions fondamentales et éthiques, la première celle de la conception de la santé de l'être humain, la deuxième celle des modalités d'échange et de coopération entre les professionnels de plus en plus mis sous pression.

En renversant le questionnement, on pourrait se demander d'ailleurs à quel niveau le travail habituel non interdisciplinaire pose des problèmes éthiques, qui peuvent potentiellement avoir des répercussions sur les patients. Ces dernières années, l'intérêt de l'approche interdisciplinaire a été souligné comme incontournable dans des articles portant sur les soins à destination de différentes populations, comme en soins palliatifs, en médecine de l'adolescence, en éducation thérapeutique, dans la prise en charge des patients précaires, handicapés etc. [5-10]. Le corollaire est qu'une formation adaptée soit proposée aux étudiants et aux 
jeunes médecins dans ce sens [11, 12] et que les instances institutionnelles soutiennent ces approches chronophages et peu rentables (en tout cas à court terme) sur le plan économique.

Notre pratique se réfère à une éthique clinique qui vise à l'amélioration de la qualité des soins offerts aux patients dans le respect de leur dignité et de leur intégrité, mais aussi de leur altérité, singularité et subjectivité. Il s'agit aussi de prendre soin de soi en même temps que des autres [13].

Nous sommes particulièrement attachés à différentes figures éthiques selon un modèle d'éthique des soins élaboré à l'UIMPV pour favoriser l'interdisciplinarité, et en tenant compte des responsabilités morales, légales, éthiques et professionnelles dans notre pratique particulière. Cette démarche prospective et dynamique doit faire sens pour les patients et les professionnels, et a pour but notamment de rétablir une identité narrative et une autonomie aux patients [14].

\section{Les registres narratifs en jeu}

\section{Prendre le temps et mettre du sens}

Notre consultation est un lieu où les personnes victimes et/ou auteurs de violences et leurs proches peuvent raconter librement, en sécurité et à leur rythme, leur perception des faits et leur vécu, et prendre le temps d'y mettre du sens et de peser les enjeux d'éventuelles décisions (dépôt de plainte, séparation, déménagement, signalement des faits aux institutions...).

La narration apparaît comme une médiation centrale du dialogue constitutif de la relation thérapeutique [15]. L'éthique narrative vise à faire en sorte que ces personnes, nourries de leur histoire propre, puissent construire une rencontre réussie. C'est une éthique de la compréhension qui demande de grands efforts, n'excuse ni n'accuse, et nécessite une conscience de la complexité humaine. Ceci implique une ouverture à autrui, une intériorisation de la tolérance, et de comprendre le rôle du conflit qui surgit suite à la confrontation des besoins, intérêts ou valeurs. Nous devons être sensibilisés à l'ambiguïté, aux ambivalences et ne pas cautionner un point de vue partiel voire partial.

De plus, la contextualisation va être une condition essentielle de l'efficacité du fonctionnement cognitif aussi bien des soignés que des soignants et donc de leur lucidité par rapport au traitement et aux autres mesures à prendre.

Cette pratique implique aussi de sortir de la position d'un thérapeute arrogant qui se place dans le contrôle et parfois l'obligation de résultat, et celle de la psycho- pathologisation de la vie qui favorise la pensée experte et disqualifie les savoirs des personnes. Il s'agit aussi d'éviter l'obsession du quantitatif, du chiffrable qui parfois est faite pour ne pas penser...

Mettre du sens aux actes et à la détresse est un objectif important de la prise en charge. Cette perspective humaniste surplombe les autres dimensions.

\section{Favoriser la relation et les liens}

La personne à soigner est tissée par des liens multiples. Un travail de soin se doit d'être réparateur, de remédiation, voire de retissage des liens. Il s'agit de soigner en

Il est important de ne pas justifier par la tolérance les intolérances des personnes qui portent les abus et injustices.

reliant la personne à elle-même, aux professionnels et à toutes les figures de résilience possibles. De même, les professionnels ne doivent pas rester seuls face aux violences et une collaboration constructive et respectueuse entre les intervenants est primordiale dans un esprit de liaison entre les savoirs et pratiques, et pour une connaissance non mutilée [16].

Les valeurs à privilégier dans la relation sont nombreuses et parfois en tension. Le patient est incité à prendre en main sa propre destinée et se responsabiliser face à ses relations. Son insertion familiale et sociale doit être favorisée.

Il s'agit aussi d'éclairer le singulier à partir du global, et le global à partir du singulier. Ainsi, la société est présente à l'intérieur de chaque individu et comprendre l'humain, c'est comprendre son unité dans la diversité, sa diversité dans l'unité [17]. C'est réinsuffler de la vie dans l'intelligence collective dans le sens où la vie n'est pas divisée en disciplines ou cultures, elle est une.

\section{Soulager et reconnaître ses limites}

C'est un objectif fondamental de l'activité soignante, avec une posture pouvant se distinguer de toute rhétorique de la guérison. Elle est censée donner un sens à l'agir soignant, valoriser et soutenir l'idéal professionnel de l'intervenant. Il s'agit de pouvoir centrer l'attention sur la personne soignée, d'instituer le soignant comme auteur dans sa pratique, de contribuer à la construction de relations soignantes. Il est important de pouvoir se laisser interpeller par l'autre et de se confronter à ses valeurs, d'entamer une démarche de questionnement, de chercher la cohérence dans son action. Dans la prise en charge et plus particulièrement dans le domaine des violences, il est conseillé de laisser la place d'une part à l'inattendu qui nous surprend et fait réviser nos théories et idées, et d'autre part à une 


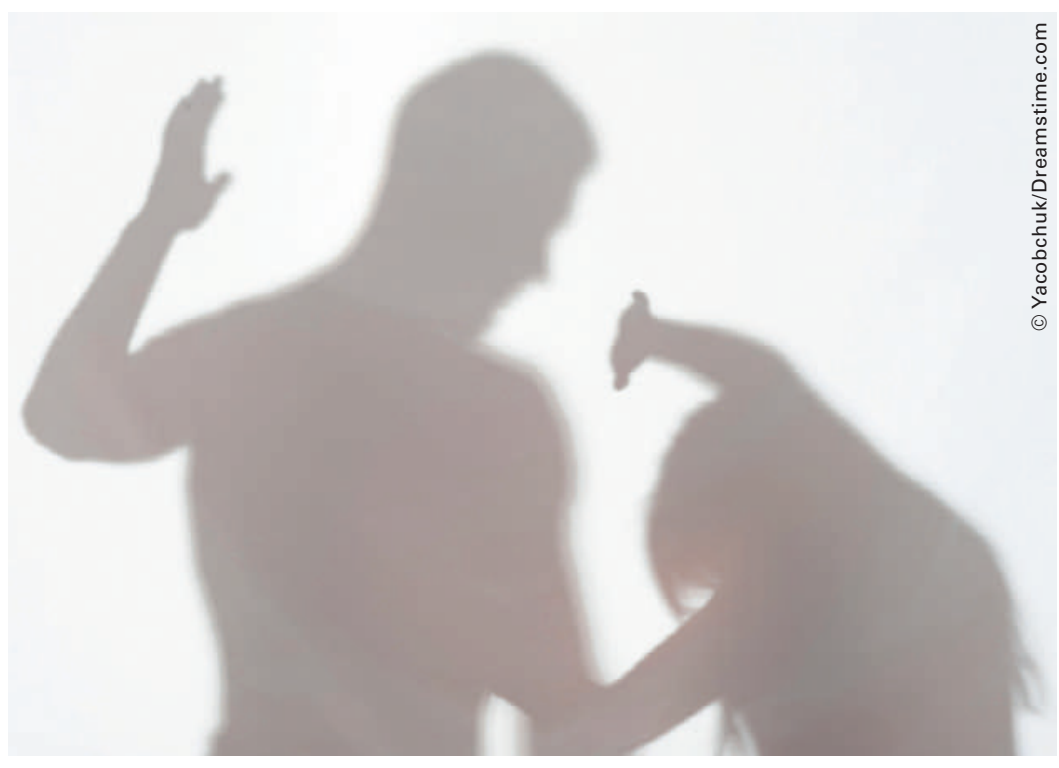

Les violences sont un problème majeur de santé publique.

conception modeste voire incertaine de la connaissance [17].

\section{Se référer à des valeurs scientifiques et pédagogiques}

Les comportements violents suscitent de nombreuses émotions et le risque est grand que des ressentis soient détournés en stéréotypes, préjugés voire dogmes qui vont avoir un impact sur nos représentations, notre pratique et la qualité des soins donnés [18]. Les violences sont éclairées par de nombreuses disciplines et les prendre en charge suppose de ne pas occulter des facteurs qui peuvent être essentiels dans leur survenue.

La prise en charge peut faire ainsi une large part aux données des sciences humaines. Une connaissance pertinente doit reconnaître la multidimensionnalité, y insérer ses données et affronter la complexité. L'écueil à éviter est de donner trop d'importance au mirage de la rationalisation qui peut atrophier la compréhension, la réflexion et la vision à long terme. La rationalité, indispensable, diffère de la rationalisation abstraite et unidimensionnelle dans le sens où elle est censée rester globale et critique [17].

Il est donc du devoir du professionnel des violences d'avoir une vision large de la problématique et de la défendre, notamment face aux nombreuses visions réductrices et aux représentations "populaires» ou médiatiques parfois délétères. Cela demande aussi un travail de vigilance et d'informations constamment renouvelé auprès de tout public, de psychoéducation des personnes victimes ou auteurs de violence et de leurs proches.

\section{Une éthique délibérative en médecine de la violence}

Les divers registres narratifs évoqués au niveau des patients, des professionnels et des projets de soins sont à mobiliser dans une approche d'éthique délibérative principale, intégrée dans cette éthique des soins spécifique [14].

\section{Rester critique et engagé}

Une des spécificités des soins dans le domaine des violences réside dans le fait que la neutralité du professionnel n'est pas de mise par rapport aux faits commis, aux réactions des patients, aux discours et représentations sur les violences. Chacun doit assumer ses responsabilités dans les risques de l'action ou de la non-action. D'où l'intérêt auparavant d'être déjà d'accord sur la définition des violences et de leurs multiples conséquences [19].

Dans la majorité des cas (chez les adultes), le patient est responsable de ses choix et capable de changement, quels que soient son comportement et sa demande. Cela implique le respect de son autonomie et de ses choix de vie, tout en le confrontant à ses responsabilités, selon les principes éthiques fondamentaux. Les comportements violents sont difficilement acceptables et justifiables, et la plupart sont sanctionnés par la loi qui est la même pour tous et fait office de tiers garant de la vie en société. Il est important de ne pas justifier par la tolérance les intolérances des personnes qui portent les abus et injustices [20]. Comprendre la diversité des comportements n'est pas les accepter de manière intégrale et inconditionnelle.

Dans un fonctionnement interdisciplinaire, les membres de l'équipe doivent s'engager à adhérer à ce modèle en vue d'atteindre un objectif commun, de bien communiquer entre eux et d'être au clair avec les rôles et responsabilités de chacun.

\section{Prendre le parti de la non-violence et de la coopération}

La coopération vise une construction en commun par le dialogue et la négociation, dans l'interactif et l'expérientiel.

Ceci est un point central de l'interdisciplinarité pour qu'elle fonctionne sainement et ne soit pas en contradiction avec un fonctionnement hiérarchique qui lui est garant des règles de fonctionnement et de l'obligation d'action et de protection en faveur des patients et de leurs proches (notamment des mineurs à charge), ni avec un certain engagement clinique.

Les compétences nécessaires pour un travail interdisciplinaire sont multiples et celui-ci demande un ap- 
prentissage et plus d'efforts constructifs au quotidien. Savoir dialoguer, gérer et dépasser les conflits et l'agressivité est par exemple capital. Cela implique de ne pas avoir peur de l'Autre, et ne pas le considérer comme un adversaire ou un rival menaçant notre propre pratique. Les compétences psychosociales sont à renforcer, notamment celles des professionnels qui ont à faire avec leur intelligence émotionnelle, relationnelle, sociale en plus de leurs compétences théoriques et techniques.

\section{Les intervenants de chaque discipline doivent être considérés à égalité dans la richesse de leurs apports.}

Les intervenants de chaque discipline doivent être considérés à égalité dans la richesse de leurs apports, ce qui implique que chacun puisse avoir son temps de parole et être respecté dans ses avis et conceptions. Chaque membre doit posséder une identité professionnelle affirmée, tout en gardant une ouverture et une souplesse permettant une remise en question régulière. Cela implique d'en finir avec les "égos disciplinaires», sources de cécité attentionnelle, et de favoriser une intelligence de groupe qui pourra être appliquée sur le terrain par le professionnel en charge du patient $[21,22]$. Celui-ci a donc un travail de coordination et de synthèse des informations pour les rendre utilisables de manière efficace.

L'enrichissement de tous passe par la loi du respect et de la solidarité plutôt que par la loi du plus fort, chacun devant rester attentif à se traiter mutuellement en sujets et non en objets.
Il s'agit en fait de l'exercice actuellement attendu de la condition humaine... Ainsi, nous tentons de nous éloigner des schémas opposés utilisés dans les mécanismes de maltraitance.

\section{En somme}

La pratique clinique, dans le domaine des violences, est d'une certaine manière indisciplinée par son exigence d'approfondissement transversal et de positionnement éthique engagé. Elle s'est adaptée pour intervenir auprès de personnes souffrant de problèmes complexes et difficiles à soulager.

La vision morcellée, réductionniste des situations qui transpirent des représentations et des pratiques aussi bien populaires que des professionnels n'est-elle pas un problème? La médecine communautaire qui peut s'exercer par le biais d'une unité interdisciplinaire a un rôle central à jouer dans la limitation de cette inadéquation entre savoirs et réalités pour le bénéfice de nos patients, de leurs proches, de leur réseau et de la société toute entière. Cela nécessite une transformation identitaire culturelle pour réduire les distances, s'émanciper de l'enfermement, donner forme et complexité à une pensée critique, reconnaître les particularismes et les principes d'universalité.

Contrairement aux actes de croyance, le fait interdisciplinaire s'impose de lui-même si l'on choisit de bien regarder en face les trajectoires humaines et les réalités vécues. Le passage à l'acte interdisciplinaire est finalement une règle d'honnêteté intellectuelle pratique, tant individuelle que collective... 